Störungen. - Bezüglich der von beiden Forschern durchgeführten Auswahl-Versuche verweise ich für den ersten Teil auf: Charlot, „Théorie et méthode nouvelle d'analyse qualitative“ Paris 1949 und für den zweiten auf die angeführte Dissertation.

Dem Verlag Walter de Gruyter danke ich für die Herausgabe des Ganzen in einer für den Gebrauch unmittelbar auf dem Laboratoriumstisch handlichen Form.

Aachen, im Sommer 1954

A. Schleicher

\title{
Vorwort des Übersetzers der 2. Auflage
}

Für die 2. Auflage war ich vor allen Dingen bemüht, die Bestimmung des Büchleins, so wie sie von Anfang an gedacht war, aufrechtzuerhalten; es soll eine Anweisung sein für den gelernten Analytiker und praktischen Chemiker, um sich auf schnellem Wege über die Zusammensetzung einer Probe an Metallen und Säureresten zu orientieren. Demgemäß konnte in ihr nicht allen Einwänden und Wünschen der Herren Kritiker der 1. Auflage nachgekommen werden. Gleichwohl aber ist die Bezeichnung dieser 2. Auflage als einer ,verbesserten und erweiterten" berechtigt. Dies kommt vor allen Dingen in dem Vorwort zur 2. Auflage der "Analyse qualitative rapide des cations" von G. CHarLor, D. Bezier und R. Gadgein, Paris 1954 zum Ausdruck. Hier heißt es: „Die Methode, so wie sie in der 1. Auflage beschrieben wurde, war insofern wenig befriedigend, als wir infolge Mangels an passenden Reaktionen gezwungen waren, zum Nachweis der Erdalkali- und Alkali-Metalle Trennungen vorzunehmen. Es mußte also die klassische Fällung aller übrigen Elemente 
in Form von Sulfiden und Hydroxyden beibehalten werden. Diese Trennung ist aber immer sehr unvollständig; es gehen beträchtliche Mengen an Erdalkali-Ionen mit dem Niederschlag verloren, und zwar durch Adsorption und Fällung als Karbonat infolge Einwirkung der Luft-Kohlensäure - und als Sulfat infolge Oxydation von Schwefelionen durch Luft-Sauerstoff. Uberdies bringt die Anwesenheit von Phosphorsäure in der Ausgangslösung und deren Abtrennung Komplikationen und zusätzliche Verluste mit sich. Daher sind die früher angegebenen Fmpfindlichkeiten illusorisch gewesen.

Durch die Einführung der Komplexone* in die analytische Praxis wird es aber möglich, die Erdalkalien sowie das Kalium ohne vorherige Abtrennungen nachzuweisen. Gleichzeitig wird aber auch der Natrium-Nachweis vereinfacht, so daß man jetzt in der Lage ist, die Erdalkali- und die häufigeren Alkali-Metalle direkt in der ursprünglichen Lösung nachzuweisen. Wenn auch die nunmehr gefundenen Empfindlichkeiten weniger gut sind als früher angegeben, so sind doch von der Gegenwart der übrigen Ionen unabhängig, was einen gewissen Vorteil bildet.

Gleichzeitig ermöglicht die Verwendung des Komplexons eine neue Charakterisierung des Quecksilbers, die ebenso eintach wie die bisher vorgeschlagene aber eine empfindlichere ist. Ebenso wurde für den $\mathrm{Kupfernachweis,} \mathrm{namentlich} \mathrm{in} \mathrm{Gegenwart} \mathrm{von} \mathrm{viel} \mathrm{Ko-}$ balt, ein neuer Weg gefunden. Er umgeht die Komplexierung des Kobalts bei der Reaktion mit Quecksilberrhodanid. Schließlich wurde eine empfindlichere Wismut- Reaktion eingeführt.

Den Einwendungen unserer Kritiker und den Erfahrungen unserer Schüler in den Einzelheiten der Durchführung der Methode wurde Rechnung getragen. Schließlich wurden auch einzelne Bezeich-

*) Komplexone sind Metallkomplexe der Aminopolycarbonsäuren, insbesondere der Äthylendiamintetraessigsäure. 
nungen geändert, wie sie in den neueren französischen und anderssprachlichen Büchern anzutreffen sind."

In den grundsätzlichen Einwendungen gegen die neue Methode ist die Frage nach der Sicherheit der Aussage aufgeworfen worden. Im systematischen Gang kann man sich diese dadurch verschaffen, daß man das Nachweisreagenz wechselt. Das ist auf dem neuen Wege noch nicht für alle Ionenarten möglich, wenn er auch einen solchen Wechsel grundsätzlich voraussieht. Beim Eisen, Molybdän, Arsen, Chrom, Quecksilber, Wismut, Kupfer, Kobalt, Zirkon, Aluminium, Zinn, Blei, Barium, Thallium und Silber sind zwei Reagenzien angegeben. Man beachte aber auch die Vorprüfungen und die zahlreichen Bemerkungen im Text über die Gruppenreagenzien, insbesondere bei den Anionen. Hier trägt jeder kleinste Hinweis zur Sicherheit der Aussage bei.

Zu den großen Fortschritten des neuen Weges rechnet aber auch die Ermittlung der Grenzkonzentrationen, denn durch sie kann man eine qualitative Aussage wesentlich genauer machen, als das bisher möglich war. Dies gilt insbesonders für diejenigen Bestandteile, welche sich dem Nachweis entzogen haben. In diesen Fällen kann man angeben, bis zu welcher Konzentration man die Suche durchgeführt hat. Nach den Erfahrungen, welche I. und W. Noddack bei der Auffindung des Rheniums gemacht haben, kann man bei genügender Anreicherung und genügend gesteigerter Empfindlichkeit des Nachweises zumindest in allen Mineralien und Erzen mit der Gegenwart aller Elemente rechnen. Es geht also nicht mehr an, bei der qualitativen Aussage lediglich die gefundenen Elemente aufzuzählen, vielmehr muß angegeben werden, auf welche Elemente man geprüft hat und bis zu welcher Konzentration man die Suche hat durchführen können.

Eine grundsätzliche Schwäche des neuen Weges ist jedoch bisher noch von keinem Kritiker gerügt worden. Der systematische An- 
alysengang wird in bezug auf die $\mathrm{p}_{\mathrm{H}}$-Werte und auf die RedoxStufe so durchgeführt, daß alle Kationen erfaßt werden können, wenn sie in der zum Nachweis genügenden Konzentration vorliegen. Es kann aber bei dem neuen Weg auch einmal vorkommen, daß bei seiner Durchführung eins der Metalle vergessen oder übersehen wird. Es gilt also auch aus diesem Grunde, die obige Forderung zu befolgen.

Der neue Weg paßt sich in seinen Empfindlichkeiten und in seinem Zeitaufwand den Fortschritten der quantitativen Analyse an, auf ihm wird die qualitative Analyse wieder zu der Vorläuferin der quantitativen; sie erleichtert diese und führt mit ihr auch zur Spurensuche.

Wir stehen somit nicht, wie eine kritische Stimme verlauten ließ, vor dem Ende der analytischen Kunst, sondern vor neuen Forschungsaufgaben eben dieser Kunst.

Beim Lesen der Korrekturen wurde ich dankenswerterweise von Frau Dr. A. Pflugmacher, Dozentin für analytische Chemie am Institut für anorganische Chemie und Elektrochemie an der Rheinisch-Westfälischen Technischen Hochschule A a chen unterstützt. 